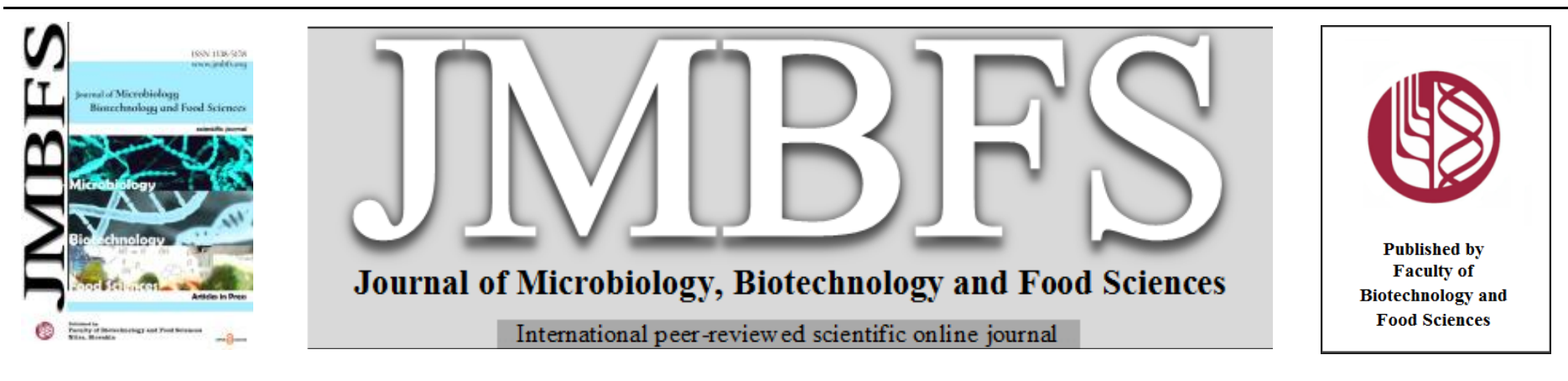

\title{
LIPASE-CATALYZED TRANSESTERIFICATION OF MEDIUM-LONG-MEDIUM STRUCTURED LIPID (MLM-SL) USING PALM OLEIN AND PALM KERNEL OIL IN BATCH AND CONTINUOUS SYSTEMS
}

\author{
Qabul Dinanta Utama ${ }^{1,3}$, Azis Boing Sitanggang ${ }^{1 *}$, Dede Robiatul Adawiyah ${ }^{1,2}$, and Purwiyatno Hariyadi,2 \\ $\operatorname{Address(es):~}$ \\ ${ }^{1}$ IPB University, Faculty of Agricultural Engineering and Technology, Department of Food Science and Technology, Darmaga, 16680 Bogor, Indonesia. \\ ${ }^{2}$ Southeast Asian Food and Agricultural Science and Technology (SEAFAST) Center, IPB University, Darmaga, 16680 Bogor, Indonesia. \\ ${ }^{3}$ University of Mataram, Faculty of Food Technology and Agroindustry, Food Science and Technology Study Program, 83115 Mataram, Indonesia.
}

*Corresponding author: boing.lipan@apps.ipb.ac.id

https://doi.org/10.55251/jmbfs.3742

ARTICLE INFO

Received 21. 9. 2020

Revised 7. 2. 2022

Accepted 8. 2. 2022

Published 1. 6. 2022

Regular article

open $\mathcal{O}$ access

\begin{abstract}
Lipase-catalyzed transesterification between refined bleached deodorized palm olein (RBDO) and palm kernel oil (RBDPKO) has been investigated to produce medium-long-medium structured lipid (MLM-SL). The synthesis was catalyzed by a specific sn-1,3 commercial lipase (Lipozyme TL IM) in batch and continuous systems. Progress of the transesterification of this study was monitored as triacylglycerol (TAG) with equivalent carbon number (ECN) 40, presumably that of 1,3-dilauryl-2-oleoyl-sn-glycerol (LaOLa). The results showed that lipase-catalyzed transesterification using RBDO and RBDPKO could potentially be used as the main substrate for MLM-SL synthesis, both for batch and continuous systems. In batch system, transesterification of RBDO and RBDPKO at the ratio of $1: 2$ at $50^{\circ} \mathrm{C}$ yielded in the highest concentration of ECN $40(\mathrm{LaLaO} / \mathrm{LaOLa}, 7.34 \%)$ but also a higher total concentration of partial acylglycerol fractions (Di- and Monoacylglycerols; DAGs and MAGs). Thus, this condition also obtained transesterified lipid rich in EC N40 with a lower slip melting point as compared to other substrate ratios. In a continuous system, transesterification at RDBO:RBDPKO of $1: 1$ at $50^{\circ} \mathrm{C}$ and 15 min of residence time were selected as the optimum conditions, resulting in $5.39 \%$ EC N40 with a minimum concentration of DAGs and MAGs.
\end{abstract}

\section{INTRODUCTION}

Lipid modification has gained attention to many researchers due to its benefit to improve physicochemical, nutritional, and functional properties of lipid. Lipid modification can be defined as a change of composition, position, or distribution of fatty acids on TAG molecule (Iwasaki \& Yamane, 2004; Lee et al., 2012). Blending, hydrogenation, chemical interesterification, and enzymatic interesterification are common methods to modify lipid. However, the enzymatic interesterification was preferably used by researchers due to its mild reaction conditions, higher selectivity (thus, less by-products), and easy recovery of catalysts (Kadhum \& Shamma, 2017; Kim \& Akoh, 2015; Samoylova et al., 2016).

One of the promising products of lipid modification is structured lipid (SL). Especially for medium-long-medium one (MLM-SL), it contains a long-chain fatty acid (LCFA, C14-C24) at $s n-2$ position and medium-chain fatty acids (MCFA, C6C12) located at $s n-1,3$ position (Adamczak \& Bornscheuer, 2013). MCFA at $s n$ 1,3 position can be used as an instant energy source due to its ability to directly transported into liver. Moreover, MCFA has a little tendency to accumulate in adipose tissue (Matulka et al., 2006; Nagao \& Yanagita, 2010). However, LCFA located at $s n-1,3$ position also show high possibility to produce calcium soap in digestive system (Karupaiah \& Sundram, 2007). This indicates the combination of MCFA and LCFA on same TAG molecule is very potential to be developed due to their nutritional benefit such as improving fat malabsorption and managing obesity (Utama et al., 2019).

In this study, refined bleached deodorized palm olein (RBDO) and palm kernel oil (RBDPKO) were used as main substrates. RBDO contains high oleic acid which was predominantly located at $s n-2$ position (Ong \& Goh, 2002; Savaghebi $\boldsymbol{e t}$ al., 2012). Oleic acid reported having positive effect on cardiovascular disease. However, RBDPKO was dominated by MCFA, especially lauric acid. Distribution of lauric acid in triacylglycerol (TAG) of RBDPKO is relatively balanced (Silalahi et al., 2018). In addition, RBDO and RBDPKO are commonly used in various food applications (Chen et al., 2007).

A specific $s n-1,3$ lipase widely used to produce high concentration of MLM-SL in transesterification reaction. Lipozyme TL IM (Novozymes A/S) is an immobilized $s n-1,3$ specific lipase produced from Thermomyces lanuginosus. The support for its immobilization is a non-compressible silica gel carrier. In addition, Lipozyme TL IM showed low economical price as compared to other commercial specific lipases (Basri et al., 2013; Wang et al., 2008; Yang et al., 2014). In our previous study, Lipozyme TL IM was more effective (i.e., obtaining higher concentration of product of interest and higher stability) to produce MLM-SL in transesterification over acidolysis reaction (Utama et al., 2020). By using this enzyme, transesterification of RBDO and RBPKO is expected to produce MLMSL with ECN 40, particularly 1,3-dilaury-2-oleoyl-sn-glycerol (LaOLa). Therefore, equivalent carbon number (ECN) 40 was chosen as TAG interest in this study.

This study aimed to synthesize MLM-SL using RBDO and RBDPKO catalyzed by Lipozyme TL IM using solvent-free system in batch and continuous systems. The effect of different mol ratios, reaction times, and residence times was investigated The change of TAG composition, acylglycerol fraction composition, and slip melting point (SMP) after transesterification were also determined.

\section{MATERIALS AND METHODS}

\section{Materials}

RBDO (iodine value/IV 60) was purchased from PT. Salim Ivomas TBK, Indonesia. RBDPKO was obtained from PT. Smart Tbk, Indonesia. Lipozyme TL IM (250 IUN/g) was purchased from Novozyme A/S, Denmark. Solvents, such as ethanol, acetone, acetonitrile chloroform, heptane, and hexane were purchased from Merck, Germany. Triglyceride standard mixture (trilaurin, tricaprin, tricaprylin, tripalmitin, and trimyristin) was purchased from Sigma-Aldrich, Singapore.

\section{Transesterification in batch system}

A total of $15 \mathrm{~g}$ of bi-substrate (RBDO and RBDPKO at different substrates mol ratio $1: 1 ; 1: 2 ; 2: 1)$ was placed in $50 \mathrm{~mL}$ Erlenmeyer flask. Lipozyme TL IM (10\% w/w) was added into reaction mixture. The reactions were carried out in solventfree system for $0,2,4,16$, and $24 \mathrm{~h}$, shaken at $200 \mathrm{rpm}$ at $50^{\circ} \mathrm{C}$. After reaction, the structured lipid product was directly filtered (Whatman No. 4, WHA1004125 Sigma-Aldrich) to separate the enzymes. The products were stored in freezer ( $<$ $-4^{\circ} \mathrm{C}$ ) for further analyses. 
Transesterification in continuous system (packed bed reactor)

The packed bed reactor system was based on our previous work (Utama et al., 2020b). Packed bed reactor ( $\mathrm{ID}=11 \mathrm{~mm}, \mathrm{H}=80 \mathrm{~mm}$ ) equipped with jacketed column was made from glass. The upper and lower ends of cylinder were equipped with filters. The column was packed with $4.5 \mathrm{~g}$ of Lipozyme TL IM. The mixture of substrate (RBDO and RBDPKO with mol ratio $1: 1 ; 1: 2$, and 2:1) flowed from substrate reservoir into packed bed reactor. Three different residence times $(15,45$ and $120 \mathrm{~min}$ ) were employed in this system. The residence time was calculated according to Equation 1 (Levenspiel, 1999; Sitanggang, Drews, \& Kraume, 2014, 2016).

$\tau=\frac{\mathrm{V}}{\mathrm{v}_{0}}$

where $\tau$ is the residence time ( $\mathrm{sec}), \mathrm{V}$ and $\mathrm{v}_{0}$ are the working volume of the reactor $\left(\mathrm{m}^{3}\right)$ and volumetric flow rate $\left(\mathrm{m}^{3} / \mathrm{s}\right)$, respectively. Temperature at substrate tank and reactor reaction were maintained at $50^{\circ} \mathrm{C}$. Sample was taken from product reservoir after $3 \mathrm{~h}$ of reaction. Each experiment was started with a fresh enzyme and bi-substrate.

\section{TAG composition analysis}

The TAG composition was analyzed using a Hewlett Packed Series 1100 HPLC system equipped with a refractive index detector (RID), Agilent Technologies, USA (Utama et al., 2020b). Mobile phase included a mixture of acetone and acetonitrile $(85: 15 \mathrm{v} / \mathrm{v})$ at a flow rate of $0.8 \mathrm{~mL} \mathrm{~min}^{-1}$. Before injection, $0.05 \mathrm{~g}$ \pm 0.005 of the sample was diluted using acetone. The injection volume included 20 $\mu 1$ and the percentage area of each peak was monitored for $60 \mathrm{~min}$. The individua TAG peak was identified based on TAG mixture standard peaks and their corresponding ECNs. ECN was calculated as $\mathrm{CN}-2(\mathrm{DB})$, where the $\mathrm{CN}$ was the total amount of carbon in the TAG molecule without glycerol and the DB was the number of double bonds on the TAG molecule (Holčapek et al., 2005)

\section{Acylglycerol fraction analysis}

The acylglycerol fractions were analyzed by means of a Hewlett Packed Series 6890 autoinjector gas chromatography system (Utama et al., 2020b). A DB-5HT column $(\mathrm{L}=15 \mathrm{~m}, \mathrm{ID}=320 \mathrm{~nm}$, and thickness $=0.1 \mu \mathrm{m})$ was used and coupled with flame ionization detector (FID) for monitoring the peaks. The complete procedures were according to AOCS Official Method Cd 11b-91 (AOCS, 2017b). The sample $(0.0250-0.0255 \mathrm{~g})$ was added with $10 \mu \mathrm{L}$ of tetrahydrofuran and $50 \mu \mathrm{L}$ of N-methyl-N-trimethylsilyl-trifluoroacetamide and vortexed at $2400 \mathrm{rpm}$ for 90 $\mathrm{s}$. The test tube was placed in the dark for $10 \mathrm{~min}$. Thereafter, a $2 \mathrm{~mL}$ of heptane was added and thoroughly vortexed at $2000 \mathrm{rpm}$ for 30 s. Sample was left for 30 min at room temperature $\left(27^{\circ} \mathrm{C}\right)$ and ready for analysis

\section{Slip melting point (SMP)}

Official procedures from AOCS Official method Cc 3-25 (AOCS, 2017a) were followed to analyze sample's slip melting point (SMP). The measurement was performed in triplicate. Sample was tempered around $10 \mathrm{~mm}$ in a capillary tube at $4-10^{\circ} \mathrm{C}$ for $16 \mathrm{~h}$. The tube was slowly heated in a beaker glass filled with water as heating medium. The temperature when samples started to rise was reported as SMP.

\section{Statistical Analysis}

One-way analysis of variance (ANOVA) was performed using SPSS 20 software (IBM, USA). Additionally, Duncan posthoc test was followed to see significant difference amongt treatments.

\section{RESULTS AND DISCUSSION}

\section{TAG composition of structured lipid in batch system}

The TAG composition of RBDO, RBDPKO, and blending product (RBDO and RBDPKO) are shown in Table 1 and Figure 1a. Prior to 20 min of retention time, RBDPKO was dominated by LaLaLa (22.43\%), LaLaM (13.46\%), and CaLaLa $(9.45 \%)$. However, the dominating TAGS changed after $20 \mathrm{~min}$ of retention time which were POO $(28.81 \%)$, POP $(21.81 \%)$, and PLO $(13.49 \%)$. Blending RBDO and RBDPKO with different mol ratios $(1: 1,1: 2$, and 1:3) showed a change in TAG profile. Blending at all ratios resulted in the dominating TAGs of POO, POP and LaLaLa varied in concentration. LaLaLa (15.33\%) showed highest concentration in blending product with a high proportion of RBDPKO (Figure 2a). However, the increasing proportion of RBDO showed highest concentration of POO $(20.52 \%)$ and reduced concentration of TAG RBDPKO (Figure 3a). In addition, POO $(18.25 \%)$ was also found as the highest TAG concentration in blending product of RBDO:RBDPKO of 1:1 (Figure 4a). This condition indicates that TAG composition of RBDO was more dominant as compared to that of RBDPKO at the same mol ratio of blending. In this study, TAG of blending product used as a representation of initial TAG before transesterification reaction.

Table 1 TAG composition of structured lipid after batch-wise transesterification at different bi-substrate blending ratios

\begin{tabular}{|c|c|c|c|c|c|c|c|c|c|c|c|c|c|c|c|c|c|c|}
\hline \multirow{3}{*}{ TAG } & \multirow{3}{*}{ ECN } & \multicolumn{17}{|c|}{ Chromatogram area (\%) of RBDO:RBDPKO } \\
\hline & & \multirow{2}{*}{ RBDO } & \multirow{2}{*}{$\begin{array}{c}\text { RBDPK } \\
\mathrm{O}\end{array}$} & \multicolumn{5}{|c|}{$(1: 1)$} & \multicolumn{5}{|c|}{$(1: 2)$} & \multicolumn{5}{|c|}{$(2: 1)$} \\
\hline & & & & T0 & $\mathrm{T} 2$ & $\mathrm{~T} 4$ & $\mathrm{~T} 16$ & $\mathrm{~T} 24$ & T0 & $\mathrm{T} 2$ & $\mathrm{~T} 4$ & $\mathrm{~T} 16$ & $\mathrm{~T} 24$ & T0 & $\mathrm{T} 2$ & $\mathrm{~T} 4$ & T16 & $\mathrm{T} 24$ \\
\hline $\mathrm{CCC}$ & 24 & ND & ND & ND & 1.73 & 1.93 & 2.29 & 3.26 & ND & 1.76 & 3.20 & 5.52 & 5.66 & ND & 2.04 & 3.36 & 4.05 & 2.88 \\
\hline $\mathrm{CCCa}$ & 26 & ND & ND & ND & 1.50 & 1.53 & 1.61 & 1.88 & ND & 2.28 & 2.59 & 3.24 & 3.74 & ND & 0.86 & 0.73 & 0.85 & 1.03 \\
\hline $\mathrm{CaCaC}$ & 28 & ND & ND & ND & 0.77 & 0.83 & 0.74 & 1.02 & ND & 1.42 & 1.60 & 1.81 & 1.61 & ND & 0.52 & 0.71 & 0.82 & 0.87 \\
\hline $\mathrm{CaCaCa}$ & 30 & ND & ND & ND & 2.07 & 2.30 & 2.48 & 2.81 & ND & 2.42 & 2.76 & 3.68 & 3.97 & ND & 1.70 & 2.21 & 2.57 & 2.47 \\
\hline ClaLa & 32 & 1.54 & 7.81 & 3.10 & 1.39 & 1.45 & 1.46 & 1.72 & 4.36 & 2.18 & 1.65 & 1.98 & 2.68 & 2.21 & 1.66 & 1.72 & 1.55 & 1.88 \\
\hline CaLaLa, ClaM & 34 & ND & 9.45 & 3.72 & 2.84 & 2.95 & 3.00 & 3.44 & 5.43 & 2.08 & 2.37 & 2.40 & 2.41 & 2.85 & 3.73 & 4.49 & 5.02 & 4.97 \\
\hline LaLaLa & 36 & ND & 22.43 & 10.97 & 3.02 & 2.79 & 1.54 & 2.50 & 15.33 & 4.71 & 4.25 & 4.66 & 2.94 & 8.47 & 0.93 & 0.94 & 0.77 & 1.01 \\
\hline LaLaM & 38 & ND & 13.46 & 6.78 & 2.01 & 1.96 & 1.95 & 1.48 & 9.39 & 4.13 & 3.76 & 3.66 & 3.06 & 5.05 & 0.96 & 0.96 & 0.61 & 0.71 \\
\hline $\mathrm{LaLaO,LaOLa}$ & 40 & ND & 5.37 & 2.12 & 5.01 & 4.94 & 4.95 & 4.12 & 3.08 & 7.34 & 6.81 & 5.86 & 5.47 & 1.63 & 2.44 & 2.28 & 2.33 & 2.36 \\
\hline LaLaP, LaMM & 40 & ND & 7.20 & 3.60 & 3.97 & 3.76 & 3.70 & 3.27 & 5.13 & 5.79 & 5.37 & 4.96 & 4.40 & 3.09 & 1.75 & 1.61 & 1.73 & 1.84 \\
\hline LLM & 42 & ND & 0.82 & ND & 2.10 & 2.23 & 2.35 & 1.94 & ND & 1.95 & 1.93 & 1.76 & 1.54 & ND & 2.13 & 2.09 & 2.15 & 2.21 \\
\hline LMM, LaOM & 42 & ND & 4.17 & 1.89 & 2.34 & 2.34 & 2.31 & 1.96 & 2.43 & 2.14 & 1.86 & 1.63 & 1.43 & 0.65 & 2.59 & 2.04 & 2.07 & 2.17 \\
\hline MMM, LaPM & 42 & ND & 3.57 & 2.14 & 3.17 & 3.51 & 3.14 & 2.96 & 2.81 & 4.91 & 4.50 & 4.01 & 3.35 & 2.00 & 2.10 & 1.97 & 2.15 & 2.08 \\
\hline $\mathrm{LMO}, \mathrm{LaOO}$ & 44 & ND & 3.61 & 1.41 & 3.09 & 3.03 & 3.08 & 2.61 & 1.01 & 4.33 & 4.05 & 3.57 & 3.72 & 2.01 & 2.21 & 2.21 & 2.34 & 2.14 \\
\hline $\begin{array}{l}\text { MPL, LaOP, } \\
\text { MMO }\end{array}$ & 44 & ND & 4.51 & 1.83 & 5.37 & 5.17 & 5.64 & 4.41 & 2.25 & 5.54 & 4.98 & 4.22 & 3.58 & 0.41 & 5.39 & 4.59 & 4.87 & 4.99 \\
\hline PLL & 44 & 2.99 & 1.18 & 2.00 & 8.69 & 8.61 & 8.98 & 7.97 & 2.52 & 8.81 & 8.06 & 7.25 & 6.14 & 1.77 & 8.05 & 7.33 & 7.78 & 8.30 \\
\hline MOO,OLO & 46 & 1.88 & 1.11 & 1.62 & 3.69 & 4.49 & 4.59 & 4.59 & 1.22 & 4.34 & 4.43 & 4.21 & 3.58 & 1.83 & 2.98 & 3.00 & 3.66 & 3.56 \\
\hline MOP,PLO & 46 & 13.49 & 2.13 & 6.63 & 3.12 & 2.85 & 2.51 & 2.00 & 4.84 & 1.30 & 1.29 & 1.13 & 1.23 & 8.12 & 4.36 & 3.85 & 4.09 & 4.19 \\
\hline PLP & 46 & 9.25 & ND & 4.38 & 1.42 & 1.45 & 1.50 & 1.30 & 3.38 & 1.30 & 1.70 & 1.26 & 1.50 & 6.26 & 1.48 & 1.37 & 1.57 & 1.51 \\
\hline MPP & 46 & 0.00 & ND & 1.39 & 3.39 & 3.55 & 3.91 & 3.11 & 1.82 & 3.33 & 3.64 & 2.83 & 3.37 & 1.67 & 3.52 & 3.76 & 3.55 & 3.15 \\
\hline $\mathrm{OOO}$ & 48 & 5.13 & 1.38 & 3.42 & 2.10 & 2.24 & 2.08 & 2.56 & 2.67 & 1.12 & 1.61 & 0.88 & 0.82 & 3.35 & 2.78 & 2.97 & 2.86 & 2.86 \\
\hline $\mathrm{POO}$ & 48 & 28.81 & 2.25 & 18.25 & 6.85 & 6.21 & 5.63 & 5.26 & 13.48 & 3.15 & 2.86 & 2.82 & 2.30 & 20.52 & 9.02 & 7.48 & 8.01 & 7.85 \\
\hline POP & 48 & 21.81 & 2.14 & 13.90 & 5.94 & 5.48 & 4.61 & 5.12 & 10.14 & 2.89 & 2.54 & 2.90 & 3.06 & 16.40 & 7.80 & 6.52 & 6.93 & 6.96 \\
\hline PPP & 48 & 3.49 & ND & ND & 1.20 & 1.27 & 1.59 & 2.25 & 1.85 & 1.04 & 1.26 & 1.22 & ND & ND & 2.23 & 2.97 & 2.36 & 2.36 \\
\hline SOO & 50 & 4.19 & ND & 2.45 & 1.26 & ND & 1.31 & 1.52 & 2.18 & 0.97 & 1.27 & ND & ND & 3.28 & 2.81 & 2.38 & 1.44 & ND \\
\hline POS & 50 & 1.54 & 7.81 & 2.90 & 1.63 & 1.46 & 2.04 & 1.55 & ND & ND & ND & ND & ND & 3.66 & 2.47 & 1.83 & 2.52 & 2.25 \\
\hline Total & & 92.58 & 92.57 & 94.49 & 79.69 & 78.33 & 78.99 & 75.91 & 95.34 & 81.23 & 80.34 & 77.48 & 71.57 & 95.24 & 78.50 & 75.35 & 78.67 & 76.58 \\
\hline Other peaks & & 7.42 & 7.43 & 5.51 & 20.31 & 21.67 & 21.01 & 24.09 & 4.66 & 18.77 & 19.66 & 22.52 & 28.43 & 4.76 & 21.50 & 24.65 & 21.33 & 23.42 \\
\hline
\end{tabular}

Legend: $\mathrm{C}=$ caprylic acid, $\mathrm{Ca}=$ Capric acid, $\mathrm{L}=$ Linoleic acid, $\mathrm{La}=$ Lauric acid, $\mathrm{M}=$ Miristic acid, $\mathrm{O}=\mathrm{Oleic}$ acid, $\mathrm{P}=\mathrm{Palmitic}$ acid, $\mathrm{S}=$ Stearic acid. 

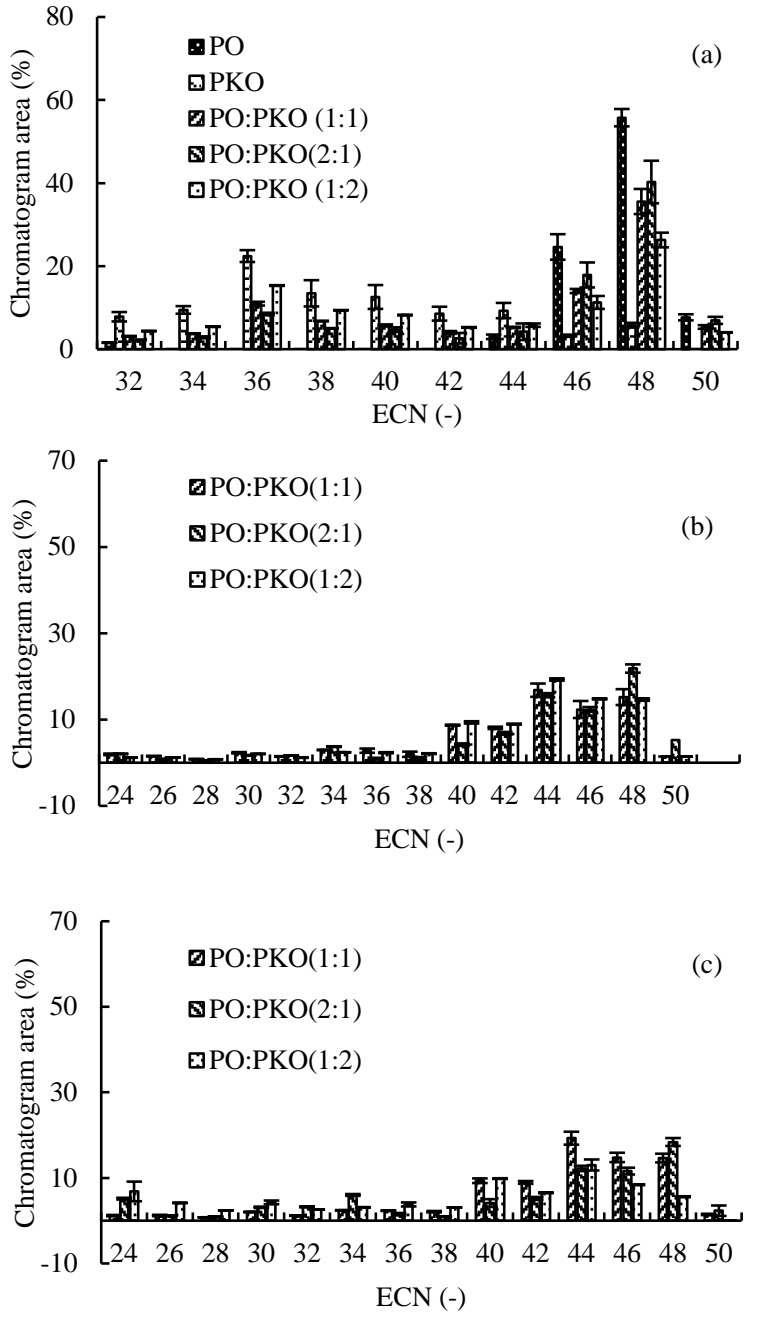

Figure 1 TAG composition based on ECN in (a) blending product, (b) batch system $(\mathrm{t}=2 \mathrm{~h})$, and $(\mathrm{c})$ continuous system $(\tau=15 \mathrm{~min})$.

The effect of different mol ratios on TAG profile in batch system transesterification reaction is shown in Table 1 and Figure $1 \mathrm{~b}$. The dominant TAGs at initial product (through blending) were depleted, leading to emergence several new TAG species. For instance, TAG species of LLM has not appeared in all blending products. However, after transesterification, LLM was detected on all transesterification products. Another potential new TAG species based on ECN are shown in Table 2. In general, different mol ratios affect the concentrations of TAGs of structured lipid product. After batch transesterification, POO, POP, and LaLaLa reduced at a higher rate especially at a mol ratio RBDO: RBDPKO of 1:1. In addition, TAGs with ECN 36, 46, and 48 were also depleted. In contrast, TAGs with ECN 40, 42, and 44 had an increase in concentration (Figure 2b). PLL was observe to have the highest increase in concentration as compared to that of other TAGs. A similar condition was also showed for RBDO:RBDPKO of 1:2 and 2:1. A higher proportion of bi-substrate showed a higher reduction of initial dominant TAGs (Table 1). POO, as a dominant TAG at blending product of RBDO:RBDPKO (2:1), showed the highest decrease during transesterification reaction. LaLaLa also showed the highest decreasing concentration at RBDO:RBDPKO of 1:2. In addition, all blending ratios also showed an increasing concentration of ECN 2430. MAGs and DAGs were also expected to increase. Chen et al. (2007) reported that transesterification reaction between palm oil and palm kernel oil produced highest interesterification degree at substrate ratio of $1: 1(\mathrm{w} / \mathrm{w})$.

In this study, the reaction time plays important role in MLM-SL synthesis Transesterification of RBDO and RBDPKO showed equilibrium condition at $2 \mathrm{~h}$ of reaction in all binary blend conditions. During $2 \mathrm{~h}$ of transesterification, POO, POP, and LaLaLa showed highest decreasing concentration. In addition, the TAG interest (i.e., LaOLa, ECN 40) showed highest concentration at $2 \mathrm{~h}$ of reaction time in all blending ratios. Therefore, reaction time of $2 \mathrm{~h}$ was selected as optimum reaction time in batch system. Longer reaction times showed a slight change in TAG profile. A longer contact time between enzyme and bi-substrate might increase the possibility of acyl migration, thus reducing the purity of transesterified product. This acyl migration was possibly due to increased water activity and support material for enzyme immobilization. Utama et al. (2020) that reported highest concentration of MLM-SL from transesterification between RBDO and tryacprylin catalyzed by Lipozyme TL IM was achieved at $4 \mathrm{~h}$ of reaction time.
Lee et al. (2013) demonstrated that $7.26 \mathrm{~h}$ of reaction was the optimum reaction time of transesterification between the palm oil and palm kernel oil

\begin{tabular}{|c|c|}
\hline ECN & TAG potential \\
\hline 24 & $\mathrm{CCC}$ \\
\hline 26 & $\mathrm{CCCa} ; \mathrm{CCaC} ; \mathrm{CaCC}$ \\
\hline 28 & $\mathrm{CaCaC} ; \mathrm{CaCCa} ; \mathrm{CCaCa} ; \mathrm{CCLa} ; \mathrm{CLaC} ; \mathrm{LaCC}$ \\
\hline 30 & $\begin{array}{l}\mathrm{CaCaCa} ; \mathrm{CaCLa} ; \mathrm{CaLaC} ; \mathrm{CLaCa} ; \mathrm{CaLaC} ; \mathrm{CCM} ; \mathrm{CMC} ; \mathrm{MCC} ; \mathrm{CCL} ; \mathrm{CLC} ; \mathrm{CCL} \\
\text {;LCC }\end{array}$ \\
\hline 32 & $\begin{array}{l}\text { CLaLa;LaLaC;LaCLa;COC;CCO;OCC;CPC;CCP;PCC;CCaM;CMCa; } \\
\text { CaMC;CaCM;MCaC;MCCa;CaCL;CaLC;CCaL;CLCa;LCaC;LCCa }\end{array}$ \\
\hline 34 & $\begin{array}{l}\text { CaLaLa;LaCLa;CLaM;CMLa;LaMC;LaCM;MCLa;MLaC;LLaC;LCLa;C } \\
\mathrm{LCa} ; \mathrm{CCaL} ; \mathrm{CaMCa} ; \mathrm{CaCaM} ; \mathrm{MCaCa} ; \mathrm{CaLCa} ; \mathrm{CaCaL} ; \mathrm{LCaCa}\end{array}$ \\
\hline 36 & $\begin{array}{l}\text { LaLaLa;CLaP;CPLa;LaCP;LaPC;PLaC;PCLa;CLaO;COLa;OLaC;OCLa; } \\
\text { LaOC;LaCO;MMC;MCM;CMM;LLC;LCL;CLL;CaML;CaLM;LCaM;L } \\
\text { MCa;MCaL;MLCa;CaLaL;CaLLa;LaCL;LaLC;LCLa;LLaC } \\
\text { LaLaM;LaMLla;MLaLa;LaLaL;LaLLa;LLaLa;CMP;CPM;MCP;MPC;PM }\end{array}$ \\
\hline 38 & $\begin{array}{l}\text { C;PCM;CaMM;MMCa;MCaM;LCaL;CaLL;LLCa;CLP;CPL;PCL;PLC;L } \\
\text { CP;LPC;SCaCa;CaCaS;CaSCa;CLO;COL;OCL;OLC;LCO;LOC }\end{array}$ \\
\hline & $\begin{array}{l}\mathrm{LaLaO} ; \mathrm{LaOLa} \text { OLaLa;LaLaP;LaPLa;PLaLa;LaLaM;LaMLa;MLaLa;LaL } \\
\text { L;LLLa;LaLLa;CaMO;CaOM;MCaO;MOCa;OCaM;OMCa;CMS;CSM;S }\end{array}$ \\
\hline 40 & $\begin{array}{l}\text { CM;SMC;MCS;MSC;CLS;CSL;LCS;LSC;SCL;SLC;CaLS;CaSL;SCaL;S } \\
\text { LCa;LCaS;LSCa;CaMP;CaPM;PCaM;PMCa;MCaP;MPCa;CaLO;CaOL;L } \\
\text { CaO;LOCa;OLCa;OCaL }\end{array}$ \\
\hline 42 & $\begin{array}{l}\text { MMM;LLL;CPS;CSP;PCS;PSC;SCP;SPC;COS;CSO;SCO;SOC;OCS;OS } \\
\text { C;CaMS;CaSM;MSCa;MCaS;SCaM;SMCa;CaLS;CaSL;LCaS;LSCa;SLC } \\
\text { a;SCaL;CaPP;PCaP;PPCa;CaOO;OCaO;OOCa;LaLaS;LaSLa;SLaLa;LaP } \\
\text { M;LaMP;MPLa;MLaP;PLaM;PMLa; }\end{array}$ \\
\hline 44 & $\begin{array}{l}\mathrm{LaOO} ; \mathrm{OLaO} ; \mathrm{OOLa} ; \mathrm{PLaP} ; \mathrm{PPL} ; \mathrm{LaPP} ; \mathrm{CSS} ; \mathrm{SCS} ; \mathrm{SSC} ; \mathrm{SCaO} ; \mathrm{SOC} ; \mathrm{CaSO} ; \\
\mathrm{CaOS} ; \mathrm{OCaS} ; \mathrm{OSCa} \text {;MMO;MOM;OMM;MMP;MPM;PMM; } \\
\text { MOO;OOM;OMO;PMP;MPP;PPM;LOO;OOL;OLO;LPP;PLL;PLP;LaPS }\end{array}$ \\
\hline 46 & $\begin{array}{l}\text {;LaSP;PSLa;PLaS;SPLa;SLaP;LaOS;LaSO;OSLa;OLaS;LaOS;LaSO;LLS } \\
\text {;LSL;SLL;MMS;MSM;SMM }\end{array}$ \\
\hline 48 & $\begin{array}{l}\text { PPP;OOO;SSLa;SLaS;LaSS;PSM;PMS;SPM;SMP;MPS;MSP;OMS;OSM } \\
\text {;SOM;SMO;MSO;MOS;LSP;LPS;SPL;SLP;PLS;PSL }\end{array}$ \\
\hline 50 & $\begin{array}{l}\text { SSM;SMS;MSS;POS;PSO;SOP;SPO;OSP;OPS;SOO;OOS;OSO;SPP;PSP } \\
\text {;PPS }\end{array}$ \\
\hline
\end{tabular}

Legend: $\mathrm{C}=$ caprylic acid, $\mathrm{Ca}=$ Capric acid, $\mathrm{L}=$ Linoleic acid, $\mathrm{La}=$ Lauric acid $\mathrm{M}=$ Miristic acid, $\mathrm{O}=$ Oleic acid, $\mathrm{P}=$ Palmitic acid, $\mathrm{S}=$ Stearic acid.
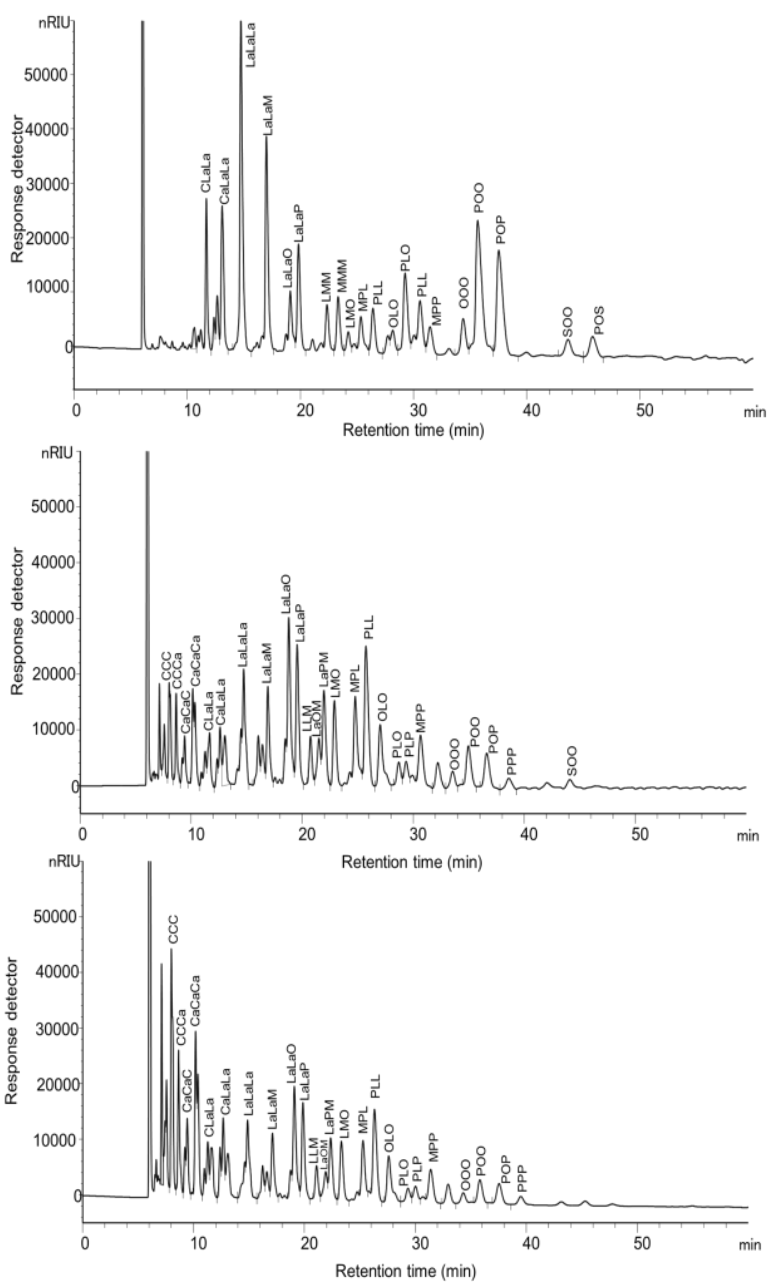

Figure 2 TAG chromatograms at RBDO:RBDPKO of 1:2. (a) blending product, (b) batch-wise $(\mathrm{t}=2 \mathrm{~h})$, and (c) continuous transesterification $(\tau=15 \mathrm{~min})$. 




Figure 3 TAG chromatograms at RBDO:RBDPKO of 2:1. (a) blending product, (b) batch-wise $(\mathrm{t}=2 \mathrm{~h})$, and (c) continuous transesterification $(\tau=15 \mathrm{~min})$.

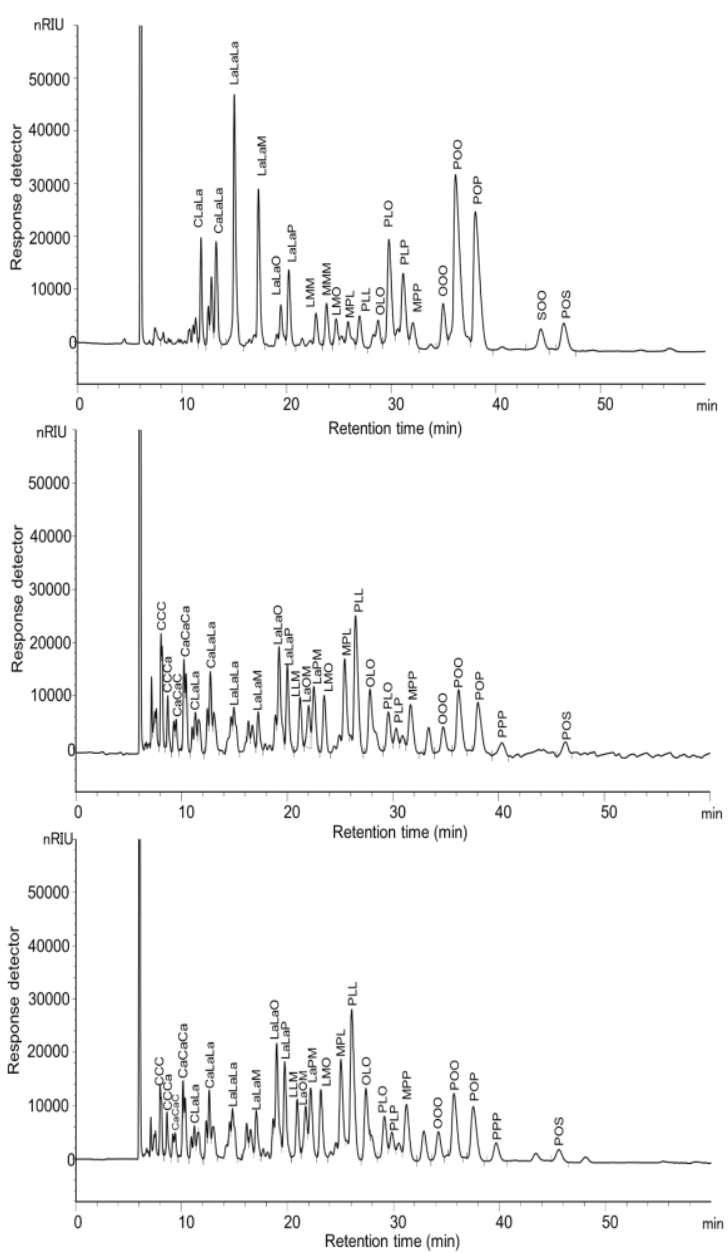

Figure 4 TAG chromatograms at RBDO:RBDPKO of 1:1. (a) blending product, (b) batch-wise $(\mathrm{t}=2 \mathrm{~h})$, and (c) continuous transesterification $(\tau=15 \mathrm{~min})$.

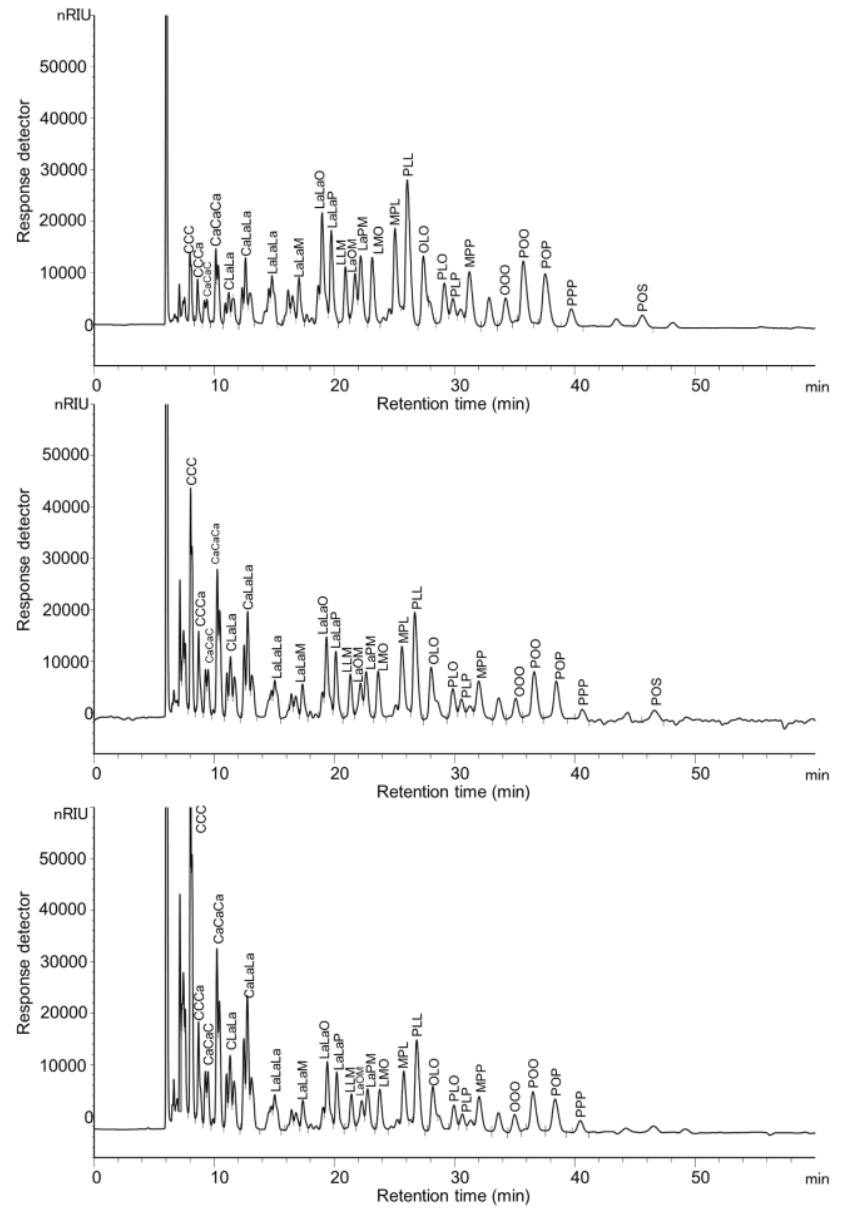

Figure 5 Effect of residence time on TAG chromatogram at RBDO:RBDPKO of $1: 1$. (a) $\tau=15 \mathrm{~min}$, (b) $\tau=30 \mathrm{~min}$, and (c) $\tau=120 \mathrm{~min}$.

\section{TAG composition of structured lipid in continuous system}

Table 3 and Figure 1c show the effect of different mol ratios on TAG profile changes during transesterification reaction in a continuous system. Based on our previous work, $15 \mathrm{~min}$ of residence time $(\tau)$ was selected as the optimum residence time to determine effect of mol ratios (Utama et al., 2020b). Similar to batch system, continuous transesterification also showed the reduction of initial dominating TAGs and emergences of new TAG species. In general, PLL had the highest concentration at all blending ratios. After transesterification, at RBDO RBDPKO of 1:1, the dominationg TAGs were PLL, POO, and $\mathrm{LaLaO} / \mathrm{LaOLa}$. A high proportion of RBDO in bi-substrate reduced POO and POP concentration. Nevertheless, POO and POP were still found as the dominating TAGs in structured lipid at RBDO: RBDPKO of 2:1. In contrast, a high proportion of RBDPKO led to a high reduction of LaLaLa concentration and yielded in structured lipid which was dominated by TAG species of CCC (ECN 24), PLL, and LaOLa. From these results, LaOLa was found dominant in two blend ratios of RBDO: RBDPKO which were 1:1 and 1:2. However, for further analysis, bi-substrate at RBDO: RBDPKO of $1: 1$ was selected as the optimal blending condition due to a higher increase in the concentration of LaOLa and lower production of acylglycerol fraction.

The blending of RBDO:RBDPKO (1:1) was used to investigate the influence of residence time on the produced TAG profiles. The transesterification was conducted at three different residence times of 15, 45, and $120 \mathrm{~min}$. From Table 4, residence time of $15 \mathrm{~min}$ showed the highest concentration of $\mathrm{LaLaO} / \mathrm{LaOLa}$ $(5.39 \%)$ as compared to that of other residence times. Longer residence time showed to reduce the concentration of LaOLa. In addition, the increasing residence time showed an increase in ECN 24 concentration. At this condition, the increasing residence time might be expected to produce MAGs and DAGs as by-product. The presence of water in reaction could facilitate hydrolysis reaction that yielded in MAGs and DAGs formation. Water availability might come from substrates, enzyme supports, or solvent used during transesterification. In this study, $15 \mathrm{~min}$ of residence time was selected as optimum condition for performing continuous reaction. Yang et al. (2014) reported that $30-40 \mathrm{~min}$ of residence time was optimum to produce MLM-SL using soybean oil medium chain triacylglycerol (MCT) catalyzed by Lipozyme TL IM in PBR system. Xu et al. (2002) also reported Lipozyme TL IM-catalyzed transesterification between fish oil and MCT in PBR system with an optimum residence time between 30-40 min. Lai et al. (2005) reported that acidolysis reaction between RBD palm olein and caprylic acid in PBR was successfully to incorporate $30.5 \%$ caprylic acid into palm olein and produced MLM-SL 
Table 4 Effect of residence time $(\tau)$ on TAG composition of structured lipid in continuous transesterification.

\begin{tabular}{|c|c|c|c|c|c|}
\hline \multirow{2}{*}{ TAG } & \multirow{2}{*}{ ECN } & \multicolumn{4}{|c|}{ Residence time ( $\tau$, min) } \\
\hline & & Blending & 15 & 45 & 120 \\
\hline $\mathrm{CCC}$ & 24 & ND & 1.19 & 4.37 & 7.08 \\
\hline $\mathrm{CCCa}$ & 26 & ND & 1.22 & 2.50 & 3.03 \\
\hline $\mathrm{CaCaC}$ & 28 & ND & 0.77 & 1.36 & 1.56 \\
\hline $\mathrm{CaCaCa}$ & 30 & ND & 2.03 & 3.93 & 4.61 \\
\hline ClaLa & 32 & 3.10 & 1.19 & 2.34 & 2.67 \\
\hline CaLaLa, ClaM & 34 & 3.72 & 2.42 & 4.02 & 4.91 \\
\hline LaLaLa & 36 & 10.97 & 2.32 & 1.62 & 1.40 \\
\hline LaLaM & 38 & 6.78 & 2.11 & 1.32 & 1.32 \\
\hline $\mathrm{LaLaO}$ & 40 & 2.12 & 5.39 & 3.79 & 3.13 \\
\hline LaLaP, LaMM & 40 & 3.60 & 3.96 & 2.93 & 2.40 \\
\hline LLM & 42 & 0.00 & 2.55 & 1.95 & 1.48 \\
\hline LMM, LaOM & 42 & 1.89 & 2.59 & 1.86 & 1.43 \\
\hline MMM, LaPM & 42 & 2.14 & 3.80 & 2.66 & 2.17 \\
\hline LMO, LaOO & 44 & 1.41 & 3.45 & 2.56 & 2.14 \\
\hline MPL, LaOP, MMO & 44 & 1.83 & 5.77 & 4.22 & 3.12 \\
\hline PLL & 44 & 2.00 & 10.06 & 7.16 & 5.49 \\
\hline MOO,OLO & 46 & 1.62 & 5.23 & 4.08 & 2.83 \\
\hline MOP,PLO & 46 & 6.63 & 2.73 & 2.06 & 1.55 \\
\hline PLP & 46 & 4.38 & 1.95 & 1.34 & 1.07 \\
\hline MPP & 46 & 1.39 & 4.88 & 3.05 & 2.53 \\
\hline OOO & 48 & 3.42 & 2.50 & 1.68 & 1.19 \\
\hline POO & 48 & 18.25 & 5.58 & 4.38 & 3.21 \\
\hline POP & 48 & 13.90 & 4.93 & 3.98 & 2.98 \\
\hline PPP & 48 & nd & 1.63 & 1.38 & 1.00 \\
\hline SOO & 50 & 2.45 & ND & ND & ND \\
\hline POS & 50 & 2.90 & 1.46 & 1.76 & ND \\
\hline Total & & 94.49 & 81.70 & 72.30 & 64.31 \\
\hline Other Peak & & 5.51 & 18.30 & 27.70 & 35.69 \\
\hline
\end{tabular}

\section{Acylglycerol fraction analysis}

Water plays important role in lipase-catalyzed interesterification. High moisture content in reacting medium leads to hydrolysis over interesterification. However, the presence of a small amount of water is still required as lubricant to maintain the rigidity of enzyme (microaqueous system). The interesterification reaction might produce DAGs, MAGs, FFAs, and glycerol as by- products. In continuous transesterification, the increasing residence time led to increased concentration of DAGs, MAGs, FFAs, and glycerol at the end of reaction. Moreover, the increasing proportion of one of substrates also increased the possibility of producing byproducts. Chen et al. (2007) reported that lipase-catalyzed transesterification between RBDO and RBDPKO catalyzed by Pseudomonas sp. lipase and Rhizomucor miehei lipase. Their studies indicated that higher proportion of RBDPKO or RBDO produced higher hydrolysis rates. However, at the equa proportion of RBDO and RBDPKO, enzyme was expected to hydrolyze TAG from RBDO and RBDPKO at the same reaction rate. After certain level, enzyme thus re-esterified fatty acids into TAG structures.

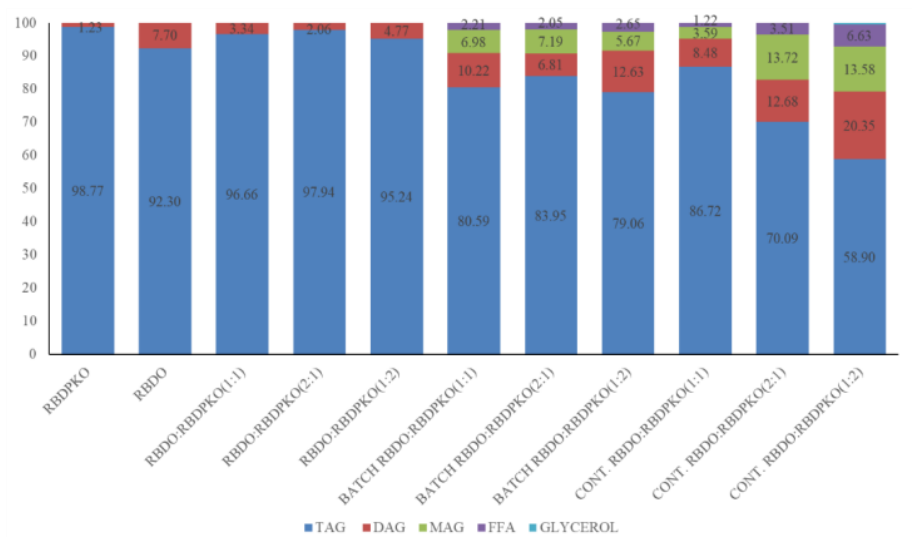

Figure 6 Acyglycerol fraction of substrates, blending products, and structured lipid product (batch-wise $t=2 \mathrm{~h}$, and continuous transesterification $\tau=15 \mathrm{~min}$ ).

Figure 6 shows that RBDO and RBDPKO were only composed by TAGs and DAGs. After the blending process, the proportion of DAGs was reduced. At RBDO:RBDPKO of 2:1, a higher total concentration of TAGs $(97.94 \%)$ was obtained as compared to that of other blending products. This indicated a high concentration of RBDO in blending product led to higher TAG concentration. After transesterification reaction either in batch or continuous system, the concentrations of MAGs, DAGs, FFAs, and glycerol increased. Zhang $\boldsymbol{e t}$ al. (2001) also reported that DAGs, MAGs, FFAs, and glycerol were by-products of transesterification, produced by a preferred hydrolysis reaction. In a batch transesterification, the highest total concentration of TAGs (83.95\%) was produced at RBDO:RBDPKO of $2: 1$. However, in a continuous reaction, the highest tota concentration of TAGs $(86.72 \%)$ was found at RBDO:RBDPKO of $1: 1$. In addition, the total concentration of TAGs in the continuous reaction was relatively higher than the batch system. This condition might be caused by different optimum conditions in batch and continuous transesterification. In a batch system, $2 \mathrm{~h}$ of reaction facilitated the bi-substrate to reproduce TAGs through the interesterification reaction. During lipase-catalyzed transesterification, a new TAG species was produced step-wise. Lipase hydrolyzed TAGs to produce DAGs and MAGs. Furthermore, between DAGs, MAGs and FAs possibly reacted again to produce a new TAG species. However, in a continuous reaction, bi-substrate had a contact time with the enzyme molecules theoretically for $15 \mathrm{~min}$. It was assumed the reaction still in a condition to produce DAGs and MAGs as intermediate products. Therefore, the total concentration of DAGs and MAGs were relatively higher in continuous transesterification rather than batc process.

\section{Slip melting point (SMP) of structured lipid}

SMP is commonly used as an indicator of physical properties of lipid. This can be used to determine future application of MLM-SL during food product development. The concentration of MAGs and DAGs may affect crystal formation, hardness of lipids thus melting point of lipids (Basso et al., 2010; Saberi $\boldsymbol{e t}$ al., 2011) In addition, types of fatty acid (length of carbon chain, presence of double bond) and isomer positions of fatty acids on DAGs and MAGs were reported to influence SMP of lipid. (Subroto et al., 2019) reported that high concentration of total saturated fatty acids in DAGs and MAGs increased melting point of lipid. In addition, Siew (2002) reported that $s n$-isomers especially 1,2 isomers of DAGs were shown to be more effective in increasing fat melting point. SMP of structured lipid product is shown in Figure 7. After transesterification, SMP was increased due to changes of acylglyerol fraction composition. Generally, the increasing of DAG concentration reduces SMP. Moreover, the increased total concentration of TAG elevates the SMP of lipid.

In this study, longer residence time in continuous transesterification produced high concentration of DAGs and MAGs which correlated to the decrease of SMP of structured lipid. A high proportion of RBDPKO fraction in bi-substrate might enhance the formation of DAGs composed of medium saturated fatty acids. This condition also led to a reduction of SMP of structured lipid. The reduction of SMP in structured lipid due to a higher protion of RBDPKO was consistent especially at RBDO:RBDPKO of 1:2 either in batch or continuous transesterification. This was corresponding to (Norizzah et al. (2018) that also mentioned a reduced SMP during enzymatic interesterification between palm oil and RBDPKO. In addition, at RBDO:RBDPKO of 1:1 showed higher SMP than at RBDO:RBDPKO of 2:1 This condition might be caused by the excessive concentration of RBDO that facilitated the production of DAGs and MAGs. As mentioned earlier, a high total concentration of MAGs and DAGs could lead to the reduction of SMP of structured lipid. On other hand, melting profile was also affected by the concentration of trisaturated TAG such as PPP. After transesterification, PPP was detected in structured lipid product at RBDO:RBDPKO of $1: 1$ and $2: 1$. However, PPP was decreased at RBDO:RBDPKO of 1:2. In this study, by evaluating the SMP, thus thermal properties of the produced structured lipid from RBDO and RBDPKO transesterification, the produced structured lipid showed potential application in food especially in solid form like chocolates or confectionary products.

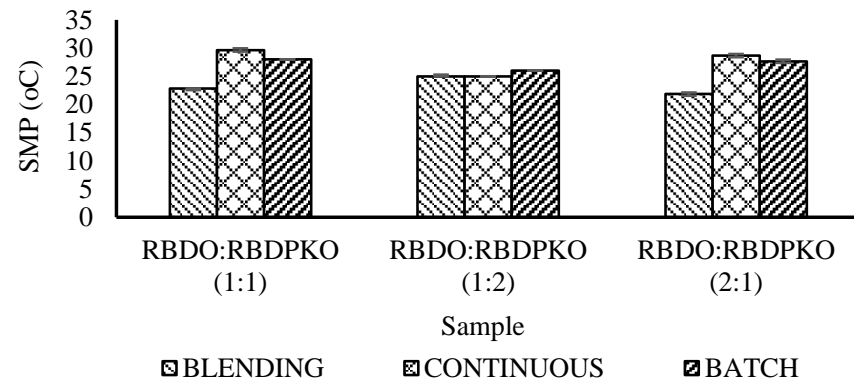

Figure 7 SMP of blending and structured lipid products (batch system $\mathrm{t}=2 \mathrm{~h}$, and continuous transesterification $\tau=15 \mathrm{~min}$ ).

\section{CONCLUSION}

RBDO and RBDPKO can potentially be used as the main substrates for producing MLM-SL, especially for TAG species of LaOLa either in batch or continuous lipase-catalyzed transesterification. In batch system, $2 \mathrm{~h}$ of reaction time and at RBDO:RBDPKO of $1: 2$ were selected as the optimum reacting conditions RBDO:RBDPKO of $1: 1$ and residence time of $15 \mathrm{~min}$ were obtained as the optimum working conditions for continuous transesterification in PBR. A higer portion of bi-substrate fraction increased the possibility to produce DAGs and MAGs that led to SMP redcution of structured lipid. 
Acknowledgements: The authors acknowledge the Ministry of Research, Technology and Higher Education of Indonesia for the financial support through The Master of Education towards Doctoral Scholarship Program for Excellent Undergraduate (PMDSU).

\section{REFERENCES}

Adamczak, M., \& Bornscheuer, U. T. (2013). Application of medium engineering in the synthesis of structured triacylglycerols from evening primrose oil (Oenothera biennis L.). European Journal of Lipid Science and Technology, 115(4), 405-412. https://doi.org/10.1002/ejlt.201200437

AOCS. (2017a). AOCS Official method Cc 3-25. In Official methods and recommended practices of the AOCS (5th ed.). Champaign, IL (USA): American Oil Chemist's Society Press.

AOCS. (2017b). AOCS Official method Cd 11b-91. In Official methods and recommended practices of the AOCS (5th ed.). Champaign, IL (USA): American Oil Chemist's Society Press.

Basri, M., Kassim, M. A., Mohamad, R., \& Ariff, A. B. (2013). Optimization and kinetic study on the synthesis of palm oil ester using Lipozyme TL im. Journal of Molecular Catalysis B: Enzymatic, 85-86, 214-219. https://doi.org/10.1016/j.molcatb.2012.09.013

Basso, R. C., Ribeiro, A. P. B., Masuchi, M. H., Gioielli, L. A., Gonçalves, L. A. G., Santos, A. O. dos, ... Grimaldi, R. (2010). Tripalmitin and monoacylglycerols as modifiers in the crystallisation of palm oil. Food Chemistry, 122(4), 1185-1192. https://doi.org/10.1016/j.foodchem.2010.03.113

Chen, C. W., Chong, C. L., Ghazali, H. M., \& Lai, O. M. (2007). Interpretation of triacylglycerol profiles of palm oil, palm kernel oil and their binary blends. Food Chemistry, 100(1), 178-191. https://doi.org/10.1016/j.foodchem.2005.09.044 Holčapek, M., Lísa, M., Jandera, P., \& Kabátová, N. (2005). Quantitation of triacylglycerols in plant oils using HPLC with APCI-MS, evaporative lightscattering, and UV detection. Journal of Separation Science, 28(12), 1315-1333. https://doi.org/10.1002/jssc.200500088

Iwasaki, Y., \& Yamane, T. (2004). Enzymatic Synthesis of Structured Lipids. Advance Biochemical Engineering/Biotechnology, 90, 151-171. https://doi.org/10.1007/b94196

Kadhum, A. A. H., \& Shamma, M. N. (2017). Edible lipids modification processes: A review. Critical Reviews in Food Science and Nutrition, 57(1), 48-58 https://doi.org/10.1080/10408398.2013.848834

Karupaiah, T., \& Sundram, K. (2007). Effects of stereospecific positioning of fatty acids in triacylglycerol structures in native and randomized fats: a review of their nutritional implications. Nutrition \& Metabolism, 4(1), 1-17. https://doi.org/10.1186/1743-7075-4-16

Kim, B. H., \& Akoh, C. C. (2015). Recent Research Trends on the Enzymatic Synthesis of Structured Lipids. Journal of Food Science, 80(8), C1713-C1724 https://doi.org/10.1111/1750-3841.12953

Lai, O. M., Low, C. T., \& Akoh, C. C. (2005). Lipase-catalyzed acidolysis of palm olein and caprylic acid in a continuous bench-scale packed bed bioreactor. Food Chemistry, 92(3), 527-533. https://doi.org/10.1016/j.foodchem.2004.08.028 Lee, Y. Y., Tang, T. K., \& Lai, O. M. (2012). Health benefits, enzymatic production, and application of medium- and long-chain triacylglycerol (mlct) in food industries: A review. Journal of Food Science, 77(8), 1-8. https://doi.org/10.1111/j.1750-3841.2012.02793.x

Lee, Y. Y., Tang, T. K., Phuah, E. T., Karim, N. A. A., Alwi, S. M. M., \& Lai, O M. (2013). Palm-based medium-and-long-chain triacylglycerol (P-MLCT) production via enzymatic interesterification and optimization using response surface methodology (RSM). Journal of Food Science and Technology, 52(2), 685-696. https://doi.org/10.1007/s13197-013-1065-0

Levenspiel, O. (1999). Chemical reaction engineering ( $3^{\text {rd }}$ ed.). New York: John Wiley \& Sons, Inc.

Matulka, R. A., Noguchi, O., \& Nosaka, N. (2006). Safety evaluation of a mediumand long-chain triacylglycerol oil produced from medium-chain triacylglycerols and edible vegetable oil. Food and Chemical Toxicology, 44(9), 1530-1538. https://doi.org/10.1016/i.fct.2006.04.004

Nagao, K., \& Yanagita, T. (2010). Medium-chain fatty acids: Functional lipids for the prevention and treatment of the metabolic syndrome. Pharmacological Research, 61(3), 208-212. https://doi.org/10.1016/j.phrs.2009.11.007

Norizzah, A. R., Nur Azimah, K., \& Zaliha, O. (2018). Influence of enzymatic and chemical interesterification on crystallisation properties of refined, bleached and deodourised (RBD) palm oil and RBD palm kernel oil blends. Food Research International, 106(2017), 982-991. https://doi.org/10.1016/j.foodres.2018.02.001 Ong, A. S.-H., \& Goh, S. (2002). Palm oil: A healthful and cost effective dietary component. Food and Nutrition Bulletin, 23(1), 11-22. https://doi.org/10.1177/156482650202300102

Saberi, A. H., Ming, L. O., \& Miskandar, M. S. (2011). Physical properties of palm-based diacylglycerol and palm-based oils in the preparation of shelf-stable margarine. European Journal of Lipid Science and Technology, 113(5), 627-636 https://doi.org/10.1002/ejlt.201000448

Samoylova, Y. V., Sorokina, K. N., \& Parmon, V. N. (2016). Prospects for application of enzymatic interesterification of oils in the production of modified fats. Catalysis in Industry, $8(4), \quad 348-353$. https://doi.org/10.1134/S2070050416040097

Savaghebi, D., Safari, M., Rezaei, K., Ashtari, P., \& Farmani, J. (2012). Structured lipids produced through lipase-catalyzed acidolysis of canola oil. Journal of Agricultural Science and Technology, 14(6), 1297-1310.

Siew, W. L. (2002). Understanding the Interactions of Diacylglycerols with Oils for Better Product Performance. Palm Oil Dev, 6-12.

Silalahi, J., Karo, L. J. K., Sinaga, S. M., \& Silalahi, Y. C. E. (2018). Composition of Fatty Acid and Identification of Lauric Acid Position in Coconut and Palm Kernel Oils. Indonesian Journal of Pharmaceutical and Clinical Research, 01(2), $1-8$.

Sitanggang, A. B., Drews, A., \& Kraume, M. (2014). Rapid transgalactosylation towards lactulose synthesis in a small-scale enzymatic membrane reactor (EMR) Chemical Engineering Transactions, 38, 19-24 https://doi.org/10.3303/CET1438004

Sitanggang, A. B., Drews, A., \& Kraume, M. (2016). Development of a continuous membrane reactor process for enzyme-catalyzed lactulose synthesis. Biochemical Engineering Journal, 109, 65-80. https://doi.org/10.1016/j.bej.2016.01.006 Subroto, E., Supriyanto, Utami, T., \& Hidayat, C. (2019). Enzymatic glycerolysisinteresterification of palm stearin-olein blend for synthesis structured lipid containing high mono- and diacylglycerol. Food Science and Biotechnology, 28(2), 511-517. https://doi.org/10.1007/s10068-018-0462-6

Utama, Q. D., Sitanggang, A. B., Adawiyah, D. R., \& Hariyadi, P. (2019). LipaseCatalyzed Interesterification for the Synthesis of Medium-Long-Medium (MLM) Structured Lipids - A Review. Food Technology \& Biotechnology, 57(3), 305318. https://doi.org/10.17113/ftb.57.03.19.6025

Utama, Q. D., Sitanggang, A. B., Adawiyah, D. R., \& Hariyadi, P. (2020a). LipaseCatalyzed Synthesis of Medium-Long-Medium-Type of Structured Lipids from Refined Bleached Deodorized Olein. Applied Food Biotechnology, 7(2), 85-94 https://doi.org/10.22037/afb.v7i2.26807

Utama, Q. D., Sitanggang, A. B., Adawiyah, D. R., \& Hariyadi, P. (2020b). Lipasecatalyzed transesterification of medium-long-medium structured lipid (MLM-SL) using palm olein and tricaprylin in packed-bed Reactor (PBR). Emirates Journal of Food and Agriculture, 32(12), 909-916 https://doi.org/10.9755/ejfa.2020.v32.i12.2209

Wang, Y., Wu, H., \& Zong, M. H. (2008). Improvement of biodiesel production by lipozyme TL IM-catalyzed methanolysis using response surface methodology and acyl migration enhancer. Bioresource Technology, 99(15), 7232-7237. https://doi.org/10.1016/j.biortech.2007.12.062

Xu, X., Porsgaard, T., Zhang, H., Adler-Nissen, J., \& Høy, C.-E. (2002). Production of structured lipids in a packed-bed reactor with thermomyces lanuginosa lipase. Journal of the American Oil Chemists' Society, 79(6), 561-565. https://doi.org/10.1007/s11746-002-0522-6

Yang, H., Mu, Y., Chen, H., Su, C., Yang, T., \& Xiu, Z. (2014). Sn-1,3-specific interesterification of soybean oil with medium-chain triacylglycerol catalyzed by lipozyme TL im. Chinese Journal of Chemical Engineering, 22(9), 1016-1020. https://doi.org/10.1016/j.cjche.2014.06.027

Zhang, H., Xu, X., Nilsson, J., Mu, H., Adler-Nissen, J., \& Høy, C. E. (2001) Production of margarine fats by enzymatic interesterification with silicagranulated thermomyces lanuginosa lipase in a large-scale study. JAOCS, Journal of the American Oil Chemists' Society, 78(1), 57-64. https://doi.org/10.1007/s11746-001-0220-4 